PAPER

\section{The neutron background of the XENON100 dark matter search experiment}

To cite this article: E Aprile et al 2013 J. Phys. G: Nucl. Part. Phys. 40115201

View the article online for updates and enhancements.
Related content

$$
\begin{aligned}
& \text { - Liquid noble gas detectors for low energy } \\
& \text { particle physics } \\
& \text { V Chepel and H Araújo } \\
& \text { - The search for particle dark matter with the } \\
& \frac{\text { XENON imaging time projection chambers }}{\text { E Aprile }} \\
& \text { - Dark matter direct-detection experiments } \\
& \text { Teresa Marrodán Undagoitia and Ludwig } \\
& \text { Rauch }
\end{aligned}
$$

\section{Recent citations}

\begin{tabular}{l} 
- DarkSide-20k: A 20 tonne two-phase LAr \\
\hline TPC for direct dark matter detection at \\
LNGS \\
C. E. Aalseth et al \\
- Effective field theory search for high- \\
$\frac{\text { energy nuclear recoils using the }}{\text { XENON100 dark matter detector }}$ \\
E. Aprile et al \\
- XENON100 dark matter results from a \\
Combination of 477 live days \\
E. Aprile et al
\end{tabular}




\title{
The neutron background of the XENON100 dark matter search experiment
}

\author{
E Aprile $^{1}$, M Alfonsi ${ }^{2}$, K Arisaka $^{3}$, F Arneodo $^{4}$, C Balan $^{5}$, L Baudis $^{6}$, \\ B Bauermeister ${ }^{7}$, A Behrens ${ }^{6}$, P Beltrame ${ }^{3,8}, \mathbf{K}_{\text {Bokeloh }}{ }^{9}, \mathbf{A}$ Brown $^{10}$, \\ E Brown ${ }^{9}$, G Bruno ${ }^{4}$, R Budnik ${ }^{1}$, J M R Cardoso ${ }^{5}$, W-T Chen ${ }^{11}$, \\ B Choi $^{1}$, A P Colijn ${ }^{2}$, H Contreras ${ }^{1}$, J P Cussonneau ${ }^{11}$, M P Decowski ${ }^{2}$, \\ E Duchovni $^{8}$, S Fattori ${ }^{7}$, A D Ferella ${ }^{4,6}$, W Fulgione $^{12}$, F Gao ${ }^{13}$, \\ M Garbini $^{14}$, C Ghag $^{3}$, K-L Giboni ${ }^{1}$, L W Goetzke ${ }^{1}$, C Grignon ${ }^{7}$, \\ E Gross $^{8}$, W Hampel ${ }^{15}$, F Kaether ${ }^{15}$, A Kish ${ }^{6,18}$, J Lamblin ${ }^{11}$,

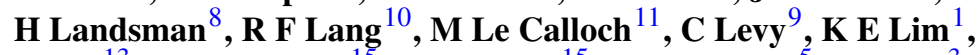 \\ Q Lin ${ }^{13}$, S Lindemann ${ }^{15}$, M Lindner ${ }^{15}$, J A M Lopes ${ }^{5}$, K Lung ${ }^{3}$, \\ T Marrodán Undagoitia ${ }^{6,15}$, F V Massoli ${ }^{14}$, A J Melgarejo Fernandez ${ }^{1}$, \\ Y Meng $^{3}$, M Messina $^{1}$, A Molinario $^{12}, \mathbf{K ~ N i}^{13}$, U Oberlack ${ }^{7}$, \\ S E A Orrigo ${ }^{5,19}$, E Pantic ${ }^{3}$, R Persiani ${ }^{14}$, G Plante ${ }^{1}$, N Priel ${ }^{8}$, A Rizzo $^{1}$, \\ S Rosendahl $^{9}$, J M F dos Santos ${ }^{5}$, G Sartorelli ${ }^{14}$, J Schreiner ${ }^{15}$, \\ M Schumann $^{6,16}$, L Scotto Lavina ${ }^{11}$, P R Scovell ${ }^{3}$, M Selvi ${ }^{14}$,

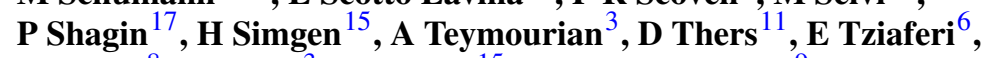

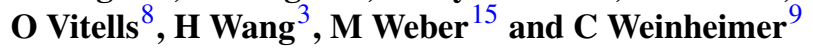 \\ ${ }^{1}$ Physics Department, Columbia University, New York, NY 10027, USA \\ ${ }^{2}$ Nikhef and the University of Amsterdam, Science Park, Amsterdam, Netherlands \\ ${ }^{3}$ Physics and Astronomy Department, University of California, Los Angeles, CA, USA \\ ${ }^{4}$ INFN, Laboratori Nazionali del Gran Sasso, Assergi, I-67100, Italy \\ 5 Department of Physics, University of Coimbra, R. Larga, 3004-516, Coimbra, Portugal \\ ${ }^{6}$ Physics Institute, University of Zürich, Winterthurerstr. 190, CH-8057, Zürich, Switzerland \\ ${ }^{7}$ Institut für Physik, Johannes Gutenberg-Universität Mainz, D-55099 Mainz, Germany \\ ${ }^{8}$ Department of Particle Physics and Astrophysics, Weizmann Institute of Science, \\ 76100 Rehovot, Israel \\ ${ }^{9}$ Institut für Kernphysik, Wilhelms-Universität Münster, D-48149 Münster, Germany \\ ${ }^{10}$ Department of Physics, Purdue University, West Lafayette, IN 47907, USA \\ ${ }^{11}$ SUBATECH, Ecole des Mines de Nantes, CNRS/In2p3, Université de Nantes, \\ F-44307 Nantes, France \\ 12 INFN-Torino and Osservatorio Astrofisico di Torino, I-10100 Torino, Italy \\ ${ }^{13}$ Department of Physics, Shanghai Jiao Tong University, Shanghai, 200240, \\ People's Republic of China \\ ${ }^{14}$ University of Bologna and INFN-Bologna, Bologna, Italy \\ ${ }^{15}$ Max-Planck-Institut für Kernphysik, Saupfercheckweg 1, D-69117 Heidelberg, Germany \\ ${ }^{16}$ Albert Einstein Center for Fundamental Physics, University of Bern, Sidlerstrasse 5, \\ 3012 Bern, Switzerland \\ ${ }^{17}$ Department of Physics and Astronomy, Rice University, Houston, TX 77005-1892, USA \\ E-mail: alexkish@physik.uzh.ch
}

Received 11 June 2013

Published 25 September 2013

Online at stacks.iop.org/JPhysG/40/115201

\footnotetext{
${ }^{18}$ Author to whom any correspondence should be addressed.

${ }^{19}$ Present address: IFIC, CSIC-Universidad de Valencia, E-46071 Valencia, Spain.
} 


\begin{abstract}
The XENON100 experiment, installed underground at the Laboratori Nazionali del Gran Sasso, aims to directly detect dark matter in the form of weakly interacting massive particles (WIMPs) via their elastic scattering off xenon nuclei. This paper presents a study on the nuclear recoil background of the experiment, taking into account neutron backgrounds from $(\alpha, n)$ reactions and spontaneous fission due to natural radioactivity in the detector and shield materials, as well as muon-induced neutrons. Based on Monte Carlo simulations and using measured radioactive contaminations of all detector components, we predict the nuclear recoil backgrounds for the WIMP search results published by the XENON100 experiment in 2011 and 2012, $0.11_{-0.04}^{+0.08}$ events and $0.17_{-0.07}^{+0.12}$ events, respectively, and conclude that they do not limit the sensitivity of the experiment.
\end{abstract}

(Some figures may appear in colour only in the online journal)

\title{
1. Introduction
}

The XENON100 detector aims at the direct detection of dark matter in the form of weakly interacting massive particles (WIMPs), and is taking data at the Laboratori Nazionali del Gran Sasso (LNGS) in Italy. It is a double phase (liquid-gas) time projection chamber (TPC) with $62 \mathrm{~kg}$ of liquid xenon (LXe) in the active volume viewed by two photomultiplier tube (PMT) arrays on the top and bottom. The design of the detector and its working principle are described in detail in [1], and the data analysis procedure is explained in [2]. To date, XENON100 is the most sensitive detector for direct dark matter detection, and has set the most stringent limits on the spin-independent elastic WIMP-nucleon scattering for WIMP masses above $8 \mathrm{GeV} / \mathrm{c}^{2}$, with a minimum cross section of $2 \times 10^{-45} \mathrm{~cm}^{2}$ at $55 \mathrm{GeV} / \mathrm{c}^{2}$ (at $90 \%$ confidence level) [3], and on the spin-dependent scattering for WIMP masses above $6 \mathrm{GeV} / \mathrm{c}^{2}$, with a minimum cross section of $3.5 \times 10^{-40} \mathrm{~cm}^{2}$ at a WIMP mass of $45 \mathrm{GeV} / \mathrm{c}^{2}$, at $90 \%$ confidence level [4].

A WIMP is expected to elastically scatter off a nucleus in the target, producing a low energy nuclear recoil (NR) [5]. There are two types of background for a dark matter search with the xenon-based detectors: NRs from hadronic interactions of neutrons, and electronic recoils (ERs) from electromagnetic interactions of $\gamma$-rays and electrons. The different ionization density characteristic of a NR and an ER results in a different probability of electron-ion pair recombination, and thus a different ratio of scintillation to ionization [6]. This provides the possibility of rejecting ER background, which is performed in XENON100 with an efficiency higher than $99 \%$ at $\sim 50 \%$ NR acceptance $[3,7,8]$.

Neutrons can produce single NRs via elastic scattering off xenon nuclei and generate a signal which is, on an event-by-event basis, indistinguishable from that of WIMPs. In addition, fast neutrons are more penetrating than $\gamma$-rays in LXe. It is therefore crucial to minimize and accurately characterize this potentially dangerous background. Due to intrinsic contamination with ${ }^{238} \mathrm{U},{ }^{235} \mathrm{U}$, and ${ }^{232} \mathrm{Th}$ of materials in the detector and shield systems, radiogenic neutrons in the $\mathrm{MeV}$ range are produced in $(\alpha, \mathrm{n})$ reactions and spontaneous fission (SF). Additionally, cosmogenic neutrons with energies extending to a few $\mathrm{GeV}$ are induced by muons penetrating through the rock into the underground laboratory. This makes the neutron yield dependent on laboratory's depth. 
A study of the ER background in the XENON100 experiment was published in [9]. In this paper we summarize results from a comprehensive Monte Carlo study, predicting the neutroninduced NR background originating from natural radioactivity and cosmic muons. The study of the radiogenic neutron background is based on calculations with the SOURCES-4A code [10]. Simulations of the cosmogenic neutron background employ the muon energy spectrum and angular distribution generated with MUSIC and MUSUN [11]. Neutron production and propagation is performed with the GEANT4 toolkit [12]. The detector model developed for the Monte Carlo simulations is described in detail in [9] and [13]. The results of this work were used to predict the neutron background in the dark matter search data acquired with XENON100 and published in $[3,7,8]$.

\section{Neutron production due to natural radioactivity}

The radiogenic neutron production rates and energy spectra were calculated with the SOURCES-4A software, modified by the group of the University of Sheffield in order to extend the cross sections for $(\alpha, \mathrm{n})$ reactions from 6.5 to $10 \mathrm{MeV}$, based on available experimental data [14]. The calculation was performed with the assumption that $\alpha$-emitters are uniformly distributed within a homogeneous material.

The systematic uncertainty on the neutron production rate of the SOURCES-4A code is $\pm 17 \%$ [10]. A cross-check of the calculations of the neutron production was performed with independent software described in [15], showing agreement in neutron rates to within $20 \%$.

The program takes into account the energy-dependent $(\alpha, \mathrm{n})$ cross sections and $Q$-values for all target nuclides, particle stopping cross sections for all elements, the energy of each $\alpha$-particle, and the SF branching ratio for each source nuclide. As an input, the code requires information about source and target nuclides, and the neutron energy range to be considered. The fractions of atoms in the target material were calculated using the chemical composition of the detector and shield components presented in table 1, and the natural isotopic abundance from [17]. The simulation takes into account all $\alpha$-emitters from each decay chain, and their half-lives, assuming secular equilibrium.

The cross section for the $(\alpha, n)$ reaction is suppressed by the Coulomb barrier for heavy nuclei, and increases with decreasing atomic number $Z$ of the target. This explains why the neutron production is dominated by $(\alpha, \mathrm{n})$ reactions for materials consisting of light elements, such as polyethylene (only $\mathrm{C}$ and $\mathrm{H}$ ) or polytetrafluoroethylene (PTFE, C and F). For high- $Z$ materials, such as copper and lead, the neutron production is almost entirely due to SF of ${ }^{238} \mathrm{U}$ (see figure 1). Thus, the neutron production rate in such materials is dependent only on the contamination level and not on the chemical composition of the material.

The neutron production rate was calculated as the number of generated neutrons over the number of disintegrating $\mathrm{U} / \mathrm{Th}$ nuclei in a given material, with the contamination of ${ }^{235} \mathrm{U}$ computed from the measured contamination of ${ }^{238} \mathrm{U}$, assuming a natural abundance of $0.72 \%$. The simulated neutron spectra for some of the materials are shown in figure 1, and the neutron production rates for all materials are presented in table 1.

In the 316Ti stainless steel (SS) used in XENON100, the secular equilibrium is broken in the ${ }^{238} \mathrm{U}$ and ${ }^{232} \mathrm{Th}$ decay chains. This was established by measuring the intrinsic radioactive contamination by inductively coupled plasma mass spectrometry performed in addition to $\gamma$-spectrometry with germanium detectors. Hence, the neutron production in this material was calculated for the different parts of the chains separately: ${ }^{238} \mathrm{U}-{ }^{230} \mathrm{Th}$ and ${ }^{226} \mathrm{Ra}-{ }^{206} \mathrm{~Pb}$, 
Table 1. Neutron production rates for the materials used in the XENON100 experiment calculated with SOURCES-4A as number of generated neutrons over number of disintegrating $\mathrm{U} / \mathrm{Th}$ nuclei. The natural abundance of ${ }^{235} \mathrm{U}$ is taken into account. The systematic uncertainty of the calculation is $\pm 17 \%$ [10]. Details on the detector and its components can be found in $[1,9]$.

Neutron production

\begin{tabular}{|c|c|c|c|c|}
\hline \multirow[b]{2}{*}{ Material } & \multirow[b]{2}{*}{ Density $\left(\mathrm{g} \mathrm{cm}^{-3}\right)$} & \multirow[b]{2}{*}{ Chemical composition } & \\
\hline & & & ${ }^{238} \mathrm{U}$ (incl. ${ }^{235} \mathrm{U}$ ) & ${ }^{232} \mathrm{Th}$ \\
\hline $\begin{array}{l}\text { Cryostat and TPC } \\
\text { 316Ti SS }\end{array}$ & 8.00 & $\begin{array}{l}\text { C } 0.08 \% \text {, Si } 1 \% \text {, Mn } 2 \% \text {, P } 0.045 \% \text {, S } 0.03 \% \text {, } \\
\text { Ni } 12 \% \text {, Cr } 17 \% \text {, Mo } 2.5 \% \text {, Ti } 4.0 \% \text {, Fe } 61.345 \%\end{array}$ & \multicolumn{2}{|c|}{ See table 2} \\
\hline $\begin{array}{l}\text { PTFE } \\
\text { Copper } \\
\text { Ceramics }\end{array}$ & $\begin{array}{l}2.20 \\
8.92 \\
1.00\end{array}$ & $\begin{array}{l}\mathrm{CF}_{2} \\
\mathrm{Cu} 100 \% \\
\mathrm{NaAlSiO}_{5}\end{array}$ & $\begin{array}{l}(6.3 \pm 1.1) \times 10^{-5} \\
(1.1 \pm 0.2) \times 10^{-6} \\
(1.1 \pm 0.2) \times 10^{-5}\end{array}$ & $\begin{array}{l}(1.0 \pm 0.2) \times 10^{-4} \\
(3.6 \pm 0.6) \times 10^{-7} \\
(2.0 \pm 0.3) \times 10^{-5} \\
\end{array}$ \\
\hline \multicolumn{5}{|l|}{ PMT parts } \\
\hline $\begin{array}{l}\text { Kovar metal } \\
\text { SS }\end{array}$ & $\begin{array}{l}8.33 \\
7.64\end{array}$ & $\begin{array}{l}\text { Fe } 55 \% \text {, Ni } 29 \% \text {, Co } 16 \% \text {; (13 g/PMT }) \\
\text { Fe } 71.8 \% \text {, C } 0.1 \% \text {, Si } 0.5 \%, \text { Mn } 0.7 \% \text {, } \\
\text { Ni } 8.6 \%, \text { Cr } 18.3 \% ;(7 \text { g/PMT })\end{array}$ & $\begin{array}{l}(1.2 \pm 0.2) \times 10^{-6} \\
(1.5 \pm 0.3) \times 10^{-6}\end{array}$ & $\begin{array}{l}(1.0 \pm 0.2) \times 10^{-6} \\
(2.3 \pm 0.4) \times 10^{-6}\end{array}$ \\
\hline Synthetic silica & 2.20 & $\mathrm{SiO}_{2} ;(2 \mathrm{~g} / \mathrm{PMT})$ & $(2.2 \pm 0.4) \times 10^{-6}$ & $(2.1 \pm 0.4) \times 10^{-6}$ \\
\hline Borosilicate glass & 2.21 & $\begin{array}{l}\mathrm{SiO}_{2} 68.7 \%, \mathrm{Al}_{2} \mathrm{O}_{3} 4.3 \%, \mathrm{~B}_{2} \mathrm{O}_{3} 18.0 \%, \\
\mathrm{Li}_{2} \mathrm{O} 1.0 \%, \mathrm{Na}_{2} \mathrm{O} 6.0 \%, \mathrm{BaO} 2.0 \% ;(1 \mathrm{~g} / \mathrm{PMT})\end{array}$ & $(1.1 \pm 0.2) \times 10^{-5}$ & $(1.5 \pm 0.3) \times 10^{-5}$ \\
\hline Aluminum & 2.70 & Al 100\%; (0.1 g/PMT) & $(1.4 \pm 0.2) \times 10^{-5}$ & $(2.8 \pm 0.5) \times 10^{-5}$ \\
\hline $\begin{array}{l}\text { Cirlex } \\
\text { Shield components }\end{array}$ & 1.43 & $\mathrm{C}_{22} \mathrm{H}_{10} \mathrm{~N}_{2} \mathrm{O}_{5} ;(1.4 \mathrm{~g} / \mathrm{PMT}$ base $)$ & $(4.8 \pm 0.8) \times 10^{-6}$ & $(2.4 \pm 0.5) \times 10^{-6}$ \\
\hline Polyethylene & 0.92 & $\mathrm{CH}_{2}$ & $(1.9 \pm 0.3) \times 10^{-6}$ & $(1.4 \pm 0.2) \times 10^{-6}$ \\
\hline $\begin{array}{l}\text { Lead } \\
\text { Environment }\end{array}$ & 11.34 & $\mathrm{~Pb} 100 \%$ & $(1.1 \pm 0.2) \times 10^{-6}$ & $(3.0 \pm 0.5) \times 10^{-11}$ \\
\hline Laboratory concrete & 2.4 & $\begin{array}{l}\text { H } 0.89 \% \text {, C } 7.99 \% \text {, O } 48.43 \% \text {, Na } 0.6 \%, \mathrm{Mg} 0.85 \% \text {, } \\
\text { Al } 0.9 \% \text {, Si } 3.86 \% \text {, P } 0.04 \% \text {, S } 0.16 \% \text {, K } 0.54 \% \text {, } \\
\text { Ca } 34.06 \% \text {, Ti } 0.04 \%, \text { Fe } 0.43 \%\end{array}$ & $(1.9 \pm 0.3) \times 10^{-6}$ & $(1.5 \pm 0.3) \times 10^{-6}$ \\
\hline
\end{tabular}




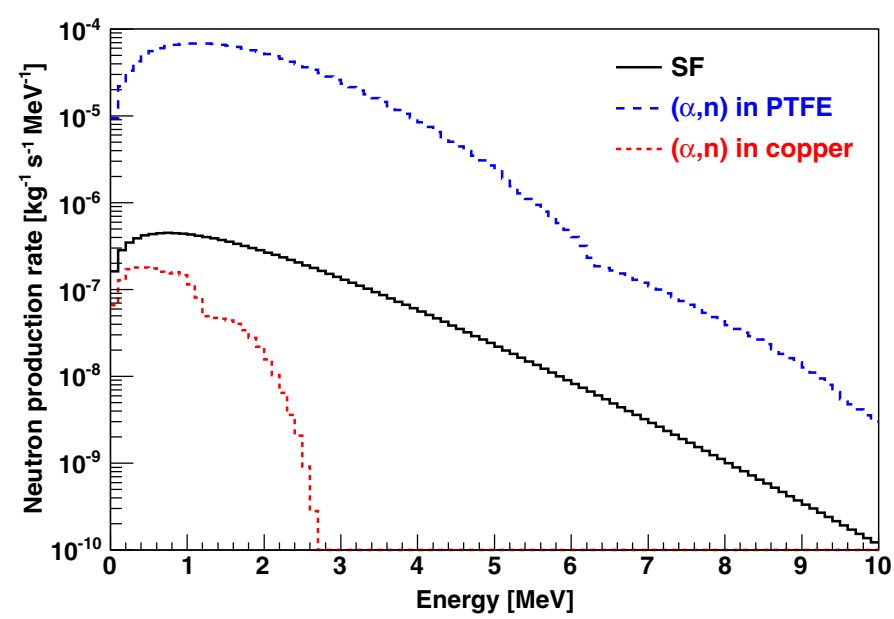

Figure 1. Differential neutron production rates in $(\alpha, n)$ and $\mathrm{SF}$ reactions in materials of the XENON100 detector and its shield due to contamination of ${ }^{238} \mathrm{U},{ }^{235} \mathrm{U}$, and ${ }^{232} \mathrm{Th}$ (assuming 1 $\mathrm{Bq}$ of ${ }^{238} \mathrm{U}$ and $1 \mathrm{~Bq}$ of ${ }^{232} \mathrm{Th}$ ). PTFE is the material with the highest production rate among the XENON100 materials. The neutron production is dominated by $(\alpha, n)$ reactions due to the low atomic mass $Z$ of elemental constituents. Copper is an example of a high $Z$ material, where the neutron production rate is almost entirely due to SF reactions. Both PTFE and copper have been used to build the TPC field cage in the XENON100 detector.

${ }^{232} \mathrm{Th}-{ }^{228} \mathrm{Ac}$ and ${ }^{228} \mathrm{Th}-{ }^{208} \mathrm{~Pb}$, and ${ }^{235} \mathrm{U}-{ }^{207} \mathrm{~Pb}$. The results are presented in table 2. Ignoring the disequilibrium, the neutron background would be underestimated.

The total neutron production rates were calculated by scaling the results of SOURCES-4A to the mass of the components in the detector and shield and to their measured radioactive contamination, using the mass model and the radioactive screening results introduced in $[9,18]$. The results are presented in table 3 .

The detector components with the highest total neutron production rates are the lead and polyethylene in the shield, and the detector cryostat and support bars made from 316Ti SS. Neutron production in the TPC resistor chain is negligible, due to the small mass involved. Even though the neutron production rate in the aluminum of the PMTs is relatively high, its contribution to the total neutron production in the PMTs is negligible due to the very low amount of material ( $0.1 \mathrm{~g}$ per PMT), since it is used only as strips deposited on the window in order to improve the resistivity of the photocathode at cryogenic temperature.

Neutron production due to natural radioactivity in the concrete walls of LNGS was calculated using the measured chemical composition of [19]. Radiogenic production in the rock has been ignored in the present study, since our simulation showed that almost all neutrons which originate in the rock are absorbed by the concrete shell. The results of our simulations agree well with the measurements of neutron flux summarized in the same paper. In particular, the discrepancy with the values measured by $[20,21]$ is less than $15 \%$.

The neutron energy spectra calculated from SOURCES-4A and the total production rates were used as an input for Monte Carlo simulations to predict the neutron-induced NR background in the XENON100 experiment. The neutron propagation was performed with GEANT4.9.3.p02, using the neutron data files G4NDL 3.13 with thermal cross sections, which are based on the ENDF/B-VI/B-VII databases [22]. For each material and neutron source, 1 million events were simulated, resulting in a negligible statistical uncertainty of $\sim 1 \%$. 
Table 2. Neutron production due to natural radioactivity in the SS (type 316Ti), mainly used for the XENON100 cryostat and its support bars. The radioactive contamination was measured with gamma- and mass-spectrometry (the most sensitive result of the two methods is given) and shows that secular equilibrium in the chains is broken.

\begin{tabular}{llllll}
\hline & ${ }^{238} \mathrm{U}-{ }^{230} \mathrm{Th}$ & ${ }^{226} \mathrm{Ra}-{ }^{206} \mathrm{~Pb}$ & ${ }^{235} \mathrm{U}-{ }^{207} \mathrm{~Pb}$ & ${ }^{232} \mathrm{Th}-{ }^{228} \mathrm{Ac}$ & ${ }^{228} \mathrm{Th}-{ }^{208} \mathrm{~Pb}$ \\
\hline Contamination $\left(\mathrm{mBq} \mathrm{kg}{ }^{-1}\right)$ & $4.9 \pm 1.6$ & $<1.3$ & $0.23 \pm 0.06$ & $<0.81$ & $2.9 \pm 0.7$ \\
Neutron production & $(1.1 \pm 0.2) \times 10^{-6}$ & $(3.1 \pm 0.5) \times 10^{-7}$ & $(4.1 \pm 0.7) \times 10^{-7}$ & $(1.8 \pm 0.3) \times 10^{-9}$ & $(2.0 \pm 0.3) \times 10^{-6}$ \\
\hline
\end{tabular}


Table 3. Neutron production rates in the XENON100 components due to $(\alpha, \mathrm{n})$ reactions and SF. Some detector components, such as the copper parts inside the cryostat, the TPC resistor chain, the bottom and top electrodes made of 316Ti SS, and PMT cables, are combined into 'additional detector parts' due to their small contribution to the total neutron production. Radioactive contamination of the laboratory concrete is taken from [16], and the neutron production is calculated as a flux.

\begin{tabular}{|c|c|c|c|c|}
\hline \multirow[b]{2}{*}{ Component } & \multirow[b]{2}{*}{ Amount } & \multicolumn{2}{|c|}{$\begin{array}{l}\text { Contamination } \\
\left(\mathrm{mBq} \mathrm{kg}^{-1}\right)\end{array}$} & \multirow[b]{2}{*}{ Neutron production (neutrons/year) } \\
\hline & & ${ }^{238} \mathrm{U}$ & ${ }^{232} \mathrm{Th}$ & \\
\hline $\begin{array}{l}\text { Cryostat and pumping } \\
\text { ports (316Ti SS) }\end{array}$ & $73.61 \mathrm{~kg}$ & \multicolumn{2}{|c|}{ see table 2} & $16 \pm 3$ \\
\hline $\begin{array}{l}\text { Cryostat support bars } \\
\text { (316Ti SS) }\end{array}$ & $49.68 \mathrm{~kg}$ & \multicolumn{2}{|c|}{ see table 2} & $13 \pm 2$ \\
\hline Detector PTFE & $11.86 \mathrm{~kg}$ & 0.06 & 0.10 & $5 \pm 1$ \\
\hline PMTs & 242 pieces & $0.05 / \mathrm{pc}$ & $0.46 / \mathrm{pc}$ & $5 \pm 1$ \\
\hline PMT bases & 242 pieces & $0.16 / \mathrm{pc}$ & $0.07 / \mathrm{pc}$ & $12 \pm 2$ \\
\hline Additional detector parts & & & & $0.20 \pm 0.03$ \\
\hline Copper shield & $2.1 \times 10^{3} \mathrm{~kg}$ & 0.083 & 0.012 & $3.2 \pm 0.5$ \\
\hline Polyethylene shield & $1.6 \times 10^{3} \mathrm{~kg}$ & 0.23 & 0.094 & $37 \pm 6$ \\
\hline Lead shield (inner layer) & $6.6 \times 10^{3} \mathrm{~kg}$ & 0.66 & 0.55 & $162 \pm 28$ \\
\hline Lead shield (outer layer) & $27.2 \times 10^{3} \mathrm{~kg}$ & 4.20 & 0.52 & $(4.3 \pm 0.7) \times 10^{3}$ \\
\hline LNGS concrete & & $2.6 \times 10^{4}$ & $8.0 \times 10^{3}$ & $(8.7 \pm 1.5) \times 10^{-7} \mathrm{~cm}^{-2} \mathrm{~s}^{-1}$ \\
\hline
\end{tabular}

In the analysis of the simulated data, only 'true' NRs in the sensitive volume were selected, meaning that all events containing an ER component have been discarded. Such a cut has a residual error, as it erroneously removes events where a Xe nucleus is excited by an inelastic neutron scatter to a relatively long-lived state, e.g. ${ }^{129 \mathrm{~m}} \mathrm{Xe}$ with $\mathrm{T}_{1 / 2}=8.9$ days, and ${ }^{131 \mathrm{~m}} \mathrm{Xe}$ with $\mathrm{T}_{1 / 2}=11.8$ days. These events have a signature of a prompt NR, followed by an ER produced by a $\gamma$ from de-excitation of the metastable state. However, by computing the ratio of the cross sections for elastic and inelastic neutron interactions with xenon nuclei, restricted to these particular nuclear levels, we estimated that the contribution of these events to the total NR rate is $<2 \%$, therefore irrelevant for the XENON100 background prediction.

Single and multiple scatters are distinguished by taking into account the ability of the XENON100 detector to separate interactions close in Z direction. It is limited to two scatters separated by more than $\sim 3 \mathrm{~mm}[1,9]$ in Z, due to the diffusion of the electron cloud and the gas gap, which define the typical width of the proportional scintillation (S2) signal. The energy spectra of NRs produced in single and multiple scatter neutron interactions are shown in figure 2 for the $34 \mathrm{~kg}$ fiducial mass used in [3].

The energy range for the WIMP search performed with 100.9 days exposure in 2011 [8] was 4-30 photoelectrons (PE). The lower threshold has been set to $3 \mathrm{PE}$ in the 2012 analysis [3] of the 224.6 days exposure, as the acceptance was still high at this value, and due to the improved electronic noise conditions. The upper threshold was kept at $30 \mathrm{PE}$ as the sensitivity does not increase significantly at higher energies. The NR energy scale is derived from the S1 signal using the relative scintillation efficiency $\mathcal{L}_{\text {eff }}$ described in [8]. Due to a change in the measured value of the LXe response to $122 \mathrm{keV}_{\text {ee }}$ gamma rays, used to normalize the scale, the energy ranges used for the 2011 and 2012 results are slightly different, and correspond to (8.4-44.6) $\mathrm{keV}_{\mathrm{nr}}$ and (6.6-43.3) $\mathrm{keV}_{\mathrm{nr}}$, respectively.

The spatial distribution of single scatter NRs in the energy region of interest (ROI) for a WIMP search is shown in figure 3. The radiogenic NR background was predicted for the entire $62 \mathrm{~kg}$ target volume and for two fiducial volumes, 48 and $34 \mathrm{~kg}$, which were used in the 


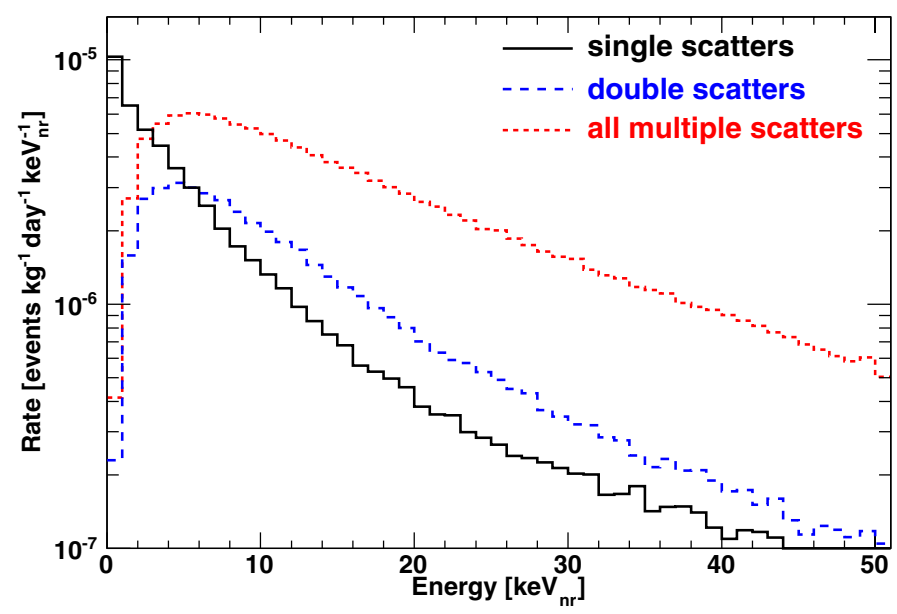

Figure 2. Energy spectra of NRs in $34 \mathrm{~kg}$ fiducial volume from neutrons produced in $(\alpha, \mathrm{n})$ and $\mathrm{SF}$ reactions. The total energy deposited in multiple scatter interactions is on average higher than that of single scatter events.

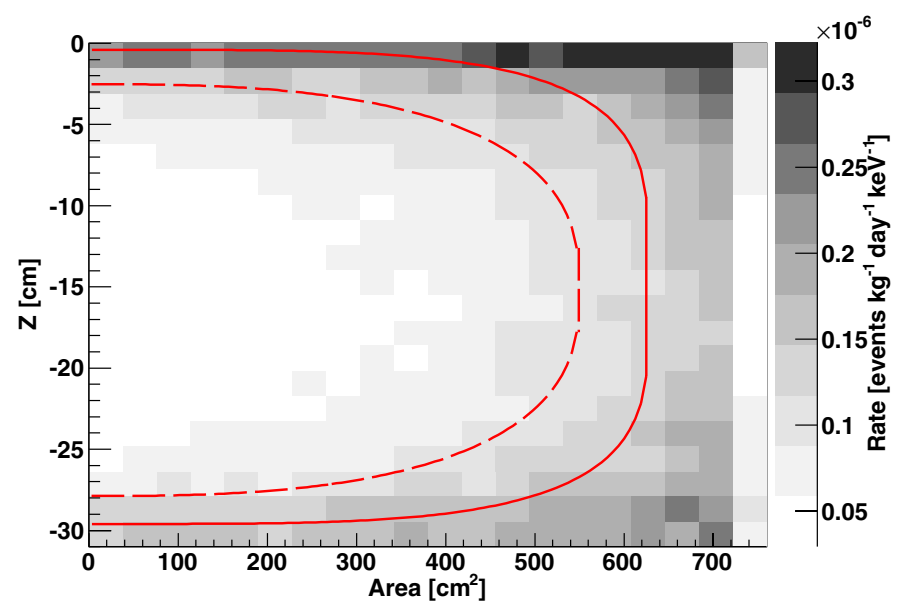

Figure 3. Spatial distribution of single scatter NRs produced by radiogenic neutrons in the energy range of interest for the WIMP search. The solid (dashed) line shows the $48 \mathrm{~kg}$ (34 kg) fiducial volume boundary. The lower density at the edge is due to the specific shape of the TPC defined by interlocking PTFE panels. This leads to a smaller active LXe volume represented by the last radial bins.

analyses published in 2011 [8] and 2012 [3], respectively. Fiducialization is less efficient for reducing the NR background because of the longer mean free path of neutrons $(\sim 14 \mathrm{~cm}$ for $1 \mathrm{MeV})$, compared to $\gamma$-rays of similar energy $(\sim 6 \mathrm{~cm})$.

The background rate of single scatter NRs in the target volume can be reduced by applying a veto coincidence cut [2], which rejects events where a particle deposits energy in the active LXe veto which surrounds the TPC. This can be either due to the same neutron which produces a NR in the target or due to an associated prompt $\gamma$-ray, for example produced by an inelastic neutron interaction. ERs and NRs in the veto volume cannot be distinguished through the ratio of scintillation and ionization signals, as it is done within the TPC. Hence, energy depositions 


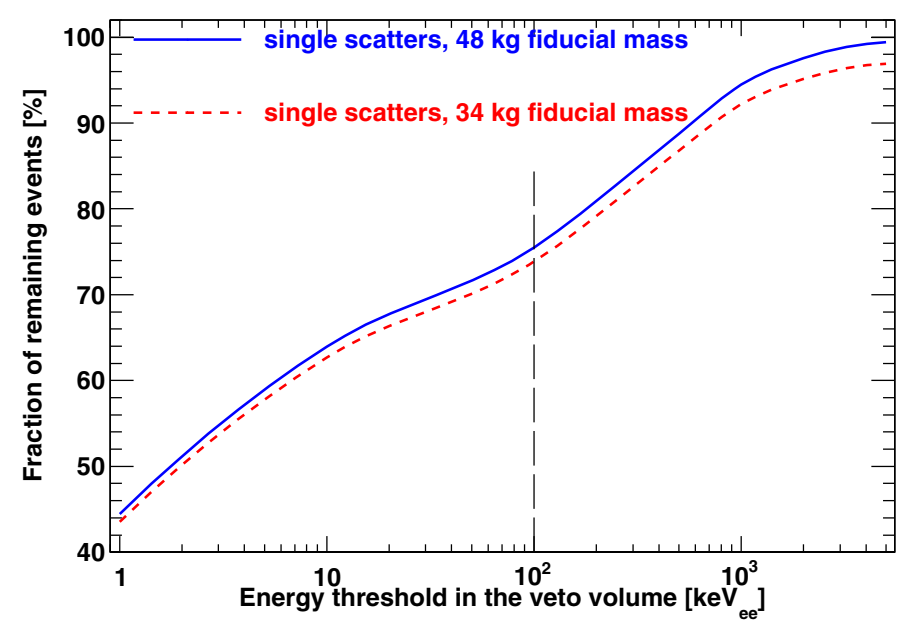

Figure 4. Efficiency of the veto coincidence cut as a function of the energy threshold in the veto volume. A veto cut with the measured volume-averaged energy threshold of $100 \mathrm{keV}_{\mathrm{ee}}$ (shown as a vertical dashed line) provides a $\sim 25 \%$ reduction of the single scatter NR rate.

Table 4. Relative contribution of different components to the total single scatter NR background due to radiogenic neutrons in XENON100. The fractions do not significantly change with the fiducialization of the target volume. 'Other components' include: polyethylene and lead shield, copper parts of the TPC, 316Ti SS support rings for the mesh electrodes, TPC resistor chain, PMT cables, and the concrete of the laboratory.

\begin{tabular}{ll}
\hline Component & Contribution (\%) \\
\hline Cryostat and pumping ports (316Ti SS) & 26 \\
Detector PTFE & 22 \\
PMTs & 21 \\
Cryostat support bars (316Ti SS) & 14 \\
PMT bases & 8 \\
Copper shield & 5 \\
Other components & 4 \\
\hline
\end{tabular}

from all interactions in the veto volume are summed up, taking into account the light quenching for NRs [8]. As shown in figure 4, by applying a cut with the measured volume-averaged energy threshold of $100 \mathrm{keV}_{\text {ee }}[1,9]$, the NR background can be reduced by $\sim 25 \%$ with respect to the 'passive veto' mode, when only self-shielding of the external LXe layer is taken into account.

The predicted NR background rates in the WIMP search region from radiogenic neutrons are presented in table 5. The contribution of the different components to the total background is shown in table 4 . The relative contributions do not significantly change by applying fiducial volume and veto coincidence cuts. The dominant part of the background comes from the $316 \mathrm{Ti}$ SS components, PMTs, and the PTFE parts of the TPC. This was expected due to the rather high neutron production rates in these components and their location close to the LXe target. Despite the high neutron production rates in the lead shield, the contribution of this source to the total NR background rate is negligible, since it is located outside of the polyethylene neutron shield. The contribution from radiogenic neutrons originating from the concrete lining of the laboratory's cavern results in $(6.6 \pm 0.4) \times 10^{-3}$ events/year in the entire target volume (62 $\mathrm{kg}$ of LXe) in the energy range of interest for the WIMP search, even without using a veto coincidence cut, and hence can be considered negligible. 

statistical error of the GEANT4 simulation is $\sim 1 \%$, hence the total error is dominated by the systematic uncertainty of the calculation with SOURCES- $4 \mathrm{~A}$. The active veto coincidence cut assumes an average detection threshold of $100 \mathrm{keV}_{\mathrm{ee}}$ in the LXe active veto. Only single scatter NRs are relevant as background for WIMP searches. The acceptance to NRs, while relevant for the WIMP search, is not taken into account here, but in section 4. The multiplicity of neutron interactions is indicated with the parameter $n$.

Predicted background rate $\left(\right.$ year $\left.^{-1}\right)$

Target volume

Energy range

$62 \mathrm{~kg}$
$8.4-44.6 \mathrm{k}$

$62 \mathrm{~kg}$

$48 \mathrm{~kg}$

$34 \mathrm{~kg}$

Veto

Passive Active

8.4-44.6 keV

Single scatter events

$\begin{array}{lll}(n=1) & 0.49 \pm 0.08 & 0.38 \pm 0.07\end{array}$

Double scatter events

Passive

Active

$34 \mathrm{~kg}$
$6.6-43.3 \mathrm{keV}$

Multiple scatter events

$\begin{array}{lll}(n=2) & 0.46 \pm 0.07 & 0.34 \pm 0.06\end{array}$

$0.28 \pm 0.05$

Passive

Active

All events

$(n>0) \quad 1.69 \pm 0.29$

$0.85 \pm 0.15$
$1.23 \pm 0.21$

$\begin{array}{lll}0.22 \pm 0.04 & 0.18 \pm 0.03 & 0.14 \pm 0.02 \\ 0.25 \pm 0.04 & 0.25 \pm 0.04 & 0.19 \pm 0.03\end{array}$

$\begin{array}{lll}0.25 \pm 0.04 & 0.25 \pm 0.04 & 0.19 \pm 0.03\end{array}$

$\begin{array}{llll}0.93 \pm 0.16 & 0.66 \pm 0.11 & 0.74 \pm 0.13 & 0.54 \pm 0.09\end{array}$

\begin{tabular}{llll}
$1.21 \pm 0.21$ & $0.88 \pm 0.15$ & $0.92 \pm 0.16$ & $0.68 \pm 0.12$ \\
\hline
\end{tabular}




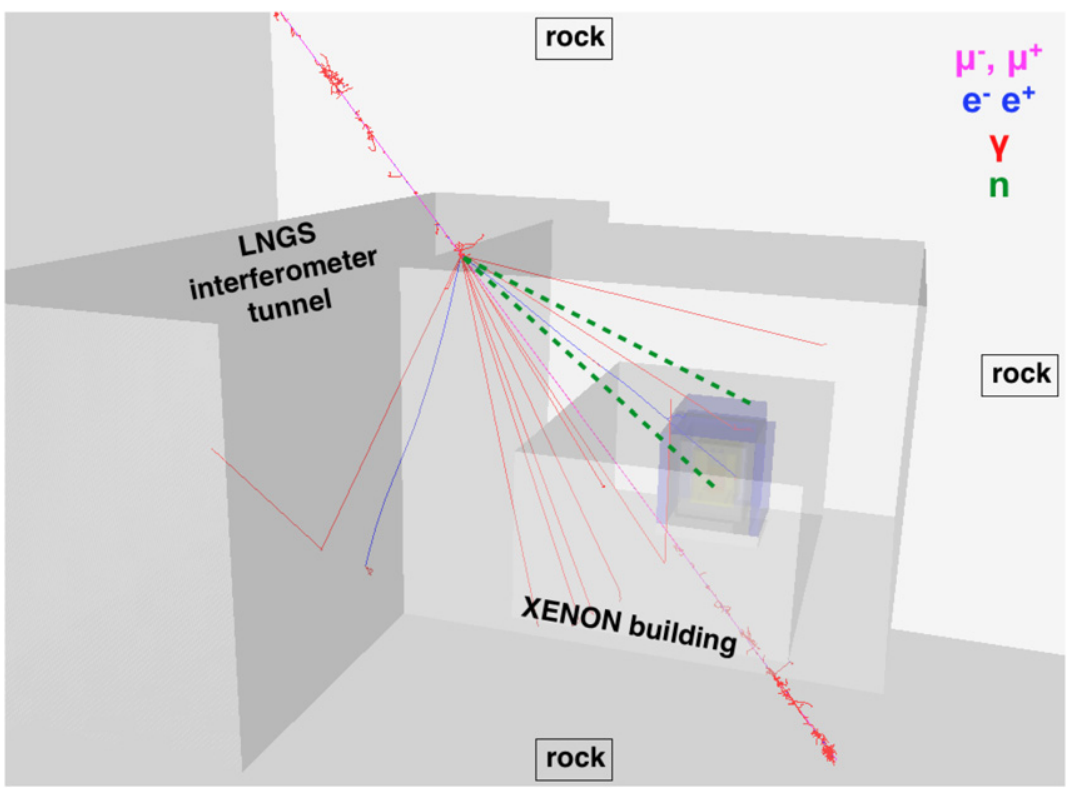

Figure 5. The GEANT4 model of the XENON100 experimental site for simulations of the cosmogenic neutron background, showing an example of a muon interaction. Two neutrons (dashed lines) and an electromagnetic shower are produced in the rock: one neutron is stopped by the shield, and another one penetrates into the detector volume.

\section{Muon-induced neutron production}

The cosmic muon flux underground at LNGS is reduced by six orders of magnitude with respect to the value measured at the surface, due to the $3.6 \mathrm{~km}$-water-equivalent, obtained averaging over the muon arrival direction, of overburden rock [23]. High-energy muons penetrating into the underground laboratory produce neutrons through photo-nuclear reactions in electromagnetic showers, in deep inelastic muon-nucleus interactions, and in several secondary processes $(\pi-n, \pi$-absorption, $p-n$, etc) $[25,26]$. The deeper the experimental site, the higher the mean muon energy and hence the average energy of muon-induced neutrons. Moreover, neutron production due to negative muon capture, which is relevant for low energy stopping muons, becomes negligible. The energy of muon-induced neutrons extends up to a few $\mathrm{GeV}$, hence the hydrocarbon and water neutron shield, as employed in XENON100, cannot fully moderate and capture them.

In order to simulate the muon-induced neutron background, the GEANT4 model introduced in [9] has been extended by including the rock and concrete lining of the underground site at LNGS, taking into account a rock thickness of $5 \mathrm{~m}$. The Gran Sasso rock is composed mainly of $\mathrm{CaCO}_{3}$ and $\mathrm{MgCO}_{3}$, and has an average density of $(2.71 \pm$ $0.05) \mathrm{g} \mathrm{cm}^{-3}$ [27]. The location of the experiment, situated in a cavity at the corner of the interferometer tunnel at LNGS, has been described with a simplified geometry. The model is shown in figure 5, together with an example of a muon interaction in the rock of the laboratory, which generates two neutrons and an electromagnetic shower: one of the neutrons is stopped by the passive shield, while the other one penetrates into the target volume.

An average muon flux of $1.2 \mathrm{~h}^{-1} \mathrm{~m}^{-2}$ was assumed for the simulations, as measured by MACRO [28] and LVD [29]. The seasonal variation of the muon intensity [31], being smaller than $2 \%$, is neglected. The muon energy spectrum and angular distribution were simulated 


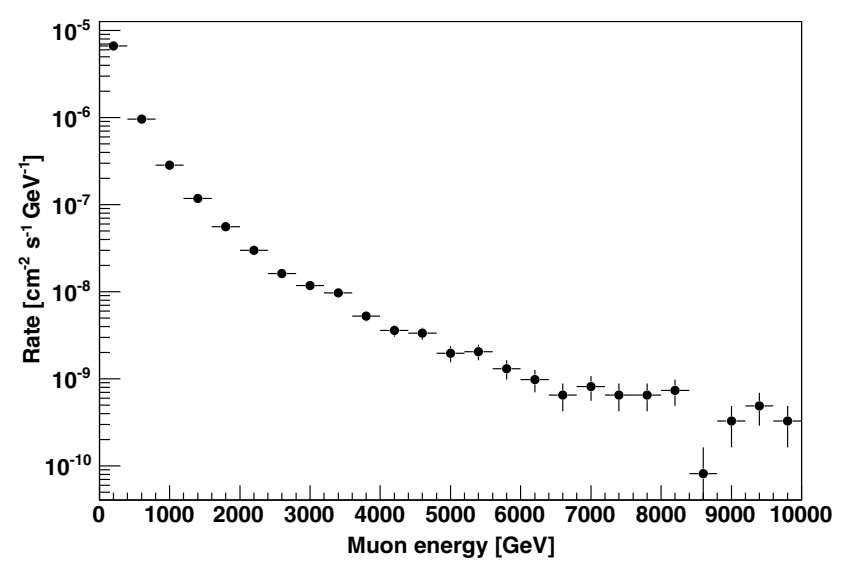

(a) energy spectrum

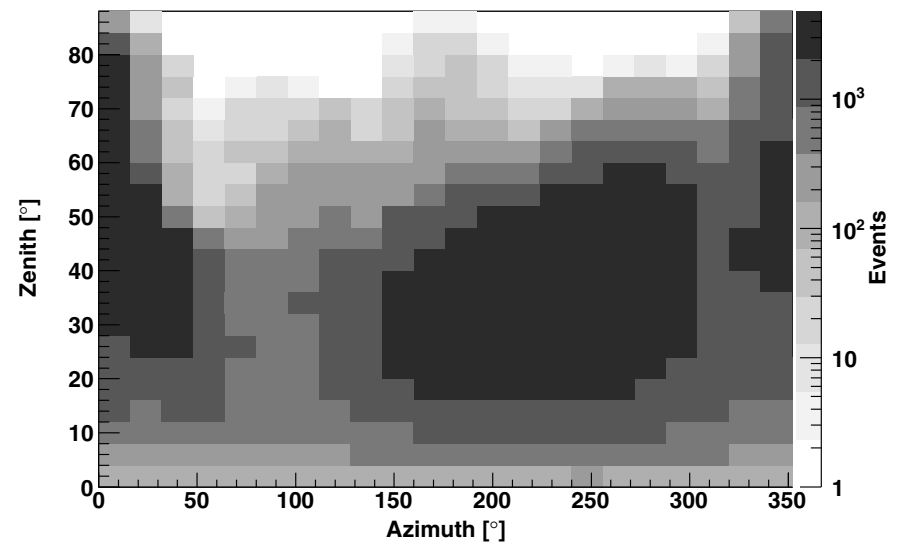

(b) angular spectrum

Figure 6. Energy and angular spectra of the muons underground at LNGS from simulations with MUSUN. The average muon energy is $273 \mathrm{GeV}$, and most of the muons have a zenith angle $<60^{\circ}$.

with the MUSIC-MUSUN package [11], taking into account the depth of the experimental hall and the details of the mountain profile; the results are shown in figure 6. The average muon energy is $273 \mathrm{GeV}$, which is in good agreement with the results in [24] and the measurements reported in [28]. The azimuthal and zenith distributions agree well with the measurement of the LVD experiment [23, 29]; there is agreement also with the predictions done with FLUKA and the measurements by MACRO and Borexino [30]. Most muon trajectories have zenith angles $<60^{\circ}$. The $\mu^{+} / \mu^{-}$ratio is assumed to be 1.4 , as shown by recent observations for high-energy muons $[32,33]$.

The propagation of the high-energy muons was performed with GEANT4.9.3.p02 using the QGSP BIC HP physics list [34], which is based on a quark gluon string model for highenergy hadronic interactions [35], and with a data-driven high-precision neutron package to transport neutrons below $20 \mathrm{MeV}$ down to thermal energies. For primary protons and neutrons with energies below $10 \mathrm{GeV}$, the GEANT4 binary cascade is used, which describes production of secondary particles in interactions with nuclei [34] more accurately than other GEANT4 models. The direct interaction between muons and nuclei is modeled with the G4MuNuclearInteraction process [36], which describes it by producing virtual photons and treating them as a combination of $\pi^{+}$and $\pi^{-}$interactions. 
Table 6. Predicted NR background rate due to muon-induced neutrons. The active veto coincidence cut assumes a volume-averaged energy threshold of $100 \mathrm{keV}_{\mathrm{ee}}$. The statistical error of the GEANT4 simulation is $\sim 10 \%$. A factor 2 systematic uncertainty in neutron production rates in GEANT4 is assumed from the comparison of simulations and measured data from the NA55 [37] and ZEPLINII $[38,39]$ experiments. The multiplicity of neutron interactions is indicated with the parameter $n$. No deficit of acceptance for NRs is considered yet (see section 4).

\begin{tabular}{|c|c|c|c|c|c|c|c|}
\hline \multirow{3}{*}{$\begin{array}{l}\text { Target volume } \\
\text { Energy range } \\
\text { Veto }\end{array}$} & & \multicolumn{6}{|c|}{ Predicted background rate $\left(\right.$ year $\left.^{-1}\right)$} \\
\hline & & \multicolumn{2}{|c|}{$\begin{array}{c}62 \mathrm{~kg} \\
8.4-44.6 \mathrm{keV}_{\mathrm{nr}}\end{array}$} & \multicolumn{2}{|c|}{$\begin{array}{c}48 \mathrm{~kg} \\
8.4-44.6 \mathrm{keV}_{\mathrm{nr}}\end{array}$} & \multicolumn{2}{|c|}{$\begin{array}{c}34 \mathrm{~kg} \\
6.6-43.3 \mathrm{keV}_{\mathrm{nr}}\end{array}$} \\
\hline & & Passive & Active & Pascive & Active & Passive & Active \\
\hline Single scatter events & $(n=1)$ & $2.2_{-1.1}^{+2.2}$ & $0.9_{-0.5}^{+0.9}$ & $1.3_{-0.6}^{+1.3}$ & $0.5_{-0.3}^{+0.5}$ & $0.9_{-0.5}^{+0.9}$ & $0.3_{-0.2}^{+0.3}$ \\
\hline Double scatter events & $(n=2)$ & $1.8_{-0.9}^{+1.8}$ & $0.7_{-0.4}^{+0.7}$ & $1.2_{-0.6}^{+1.2}$ & $0.5_{-0.3}^{+0.5}$ & $1.0_{-0.5}^{+1.0}$ & $0.3_{-0.2}^{+0.3}$ \\
\hline Multiple scatter events & $(n>1)$ & $5.6_{-2.8}^{+5.6}$ & $2.0_{-1.0}^{+2.0}$ & $4.3_{-2.2}^{+4.3}$ & $1.5_{-0.8}^{+1.5}$ & $3.4_{-1.7}^{+3.4}$ & $1.0_{-0.5}^{+1.0}$ \\
\hline All events & $(n>0)$ & $7.8_{-3.9}^{+7.8}$ & $2.9_{-1.4}^{+2.9}$ & $5.6_{-2.8}^{+5.6}$ & $2.1_{-1.0}^{+2.1}$ & $4.3_{-2.2}^{+4.3}$ & $1.4_{-0.7}^{+1.4}$ \\
\hline
\end{tabular}

Table 7. Relative contribution from the detector and shield components to the muon-induced neutron background. It is given for all neutrons that produce NRs in the target volume (left column), and also for those neutrons that have only a true single scatter NR signature in the energy ROI.

\begin{tabular}{lll}
\hline & \multicolumn{2}{c}{ Contribution (\%) } \\
\cline { 2 - 3 } Component/Material & All NRs & $\begin{array}{l}\text { Single scatter } \\
\text { NRs in ROI }\end{array}$ \\
\hline Rock and concrete & $<1$ & 5 \\
Water shield & $<1$ & 5 \\
Lead shield & 6 & 15 \\
Polyethylene shield & 2 & 5 \\
Copper shield & 33 & 55 \\
Cryostat and detector ports & 3 & $<1$ \\
Detector PTFE & 5 & 10 \\
LXe & 46 & 5 \\
Other components & 5 & $<1$ \\
\hline
\end{tabular}

About 300 million muons were simulated, corresponding to about 185 years of livetime. This results in a statistical uncertainty of $\sim 10 \%$ on the background prediction. Information from the literature was used in order to evaluate the systematic uncertainty of the simulations. The muon-induced neutron production with GEANT4 was validated in [37] via comparison with measured data from the NA55 experiment, resulting in a factor of $\sim 2$ underproduction by the Monte Carlo simulation. Such comparison has been also performed in [38, 39], using the experimental data of ZEPLIN-II, and in [40] for the KamLAND experiment. Both studies indicate a factor of $\sim 2$ overproduction for heavy targets, such as lead, by GEANT4. These results have been used to set the systematic uncertainty of the simulations for GEANT4 by assigning asymmetric error bars, which are the size of a factor \pm 2 relative to the mean value. At the same time, a new study performed by ZEPLIN-III [41] shows that more accurate results can be achieved using updated versions of GEANT4 and physics models; in this case Monte Carlo simulations result in a higher neutron production yield, which in a better agreement with the measured data. Based on these results, and taking into account the used GEANT4 versions, we expect the difference within the systematic uncertainty assumed in our study.

The background rates from muon-induced neutrons are presented in table 6, and the contribution of different detector components to the total muon-induced neutron background is given in table 7. It was calculated for all neutrons that produce NRs in the target volume, 


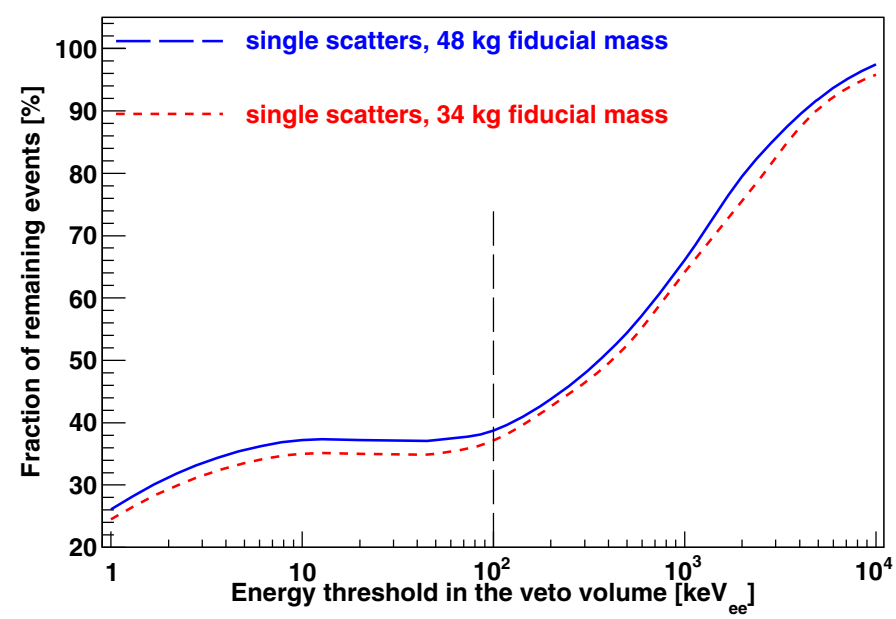

Figure 7. Efficiency of the veto coincidence cut as a function of the energy deposited in the veto volume for reduction of the cosmogenic neutron background. A veto cut with the measured volume-averaged energy threshold of $100 \mathrm{keV}_{\text {ee }}$ provides a $\sim 60 \%$ reduction of the single scatter NR rate.

and also for those neutrons that have a true single scatter NR signature and contribute to the background in the WIMP search energy ROI. The production rate of neutrons in LXe is relatively high. However, they do not contribute significantly to the NR background, as they are coincident with high-energy depositions from the primary muon or an associated electromagnetic cascade. The largest contribution (55\%) to the background is from neutrons generated in the innermost (copper) shield.

The spatial distribution of NRs due to cosmogenic neutrons is more uniform than those of radiogenic origin, due to their higher energies and therefore longer mean free path in the LXe. Therefore, fiducialization of the target volume is not efficient in reducing this background source. As detectors get larger, identification of these neutrons becomes easier through their detected multiplicity.

Since muon-induced neutron production is often accompanied by an electromagnetic cascade and by a high-energy deposition from the incident muon, this background can be reduced by applying a veto coincidence cut. As shown in figure 7, a veto coincidence cut with the measured volume-averaged threshold of $100 \mathrm{keV}_{\text {ee }}$ rejects more than half of the single scatter NRs in the target volume.

\section{Total predicted nuclear recoil background}

The Monte Carlo simulations of the NR background presented above assume $100 \%$ detection efficiency. The measured trigger efficiency is $>99 \%$ at 300 (150) PE in the 2011 (2012) WIMP search results and rolls off at lower values [1], which reduces the detection efficiency. In addition, the acceptance for single scatter events is finite, and is determined by the size of the proportional scintillation (S2) signal. If one interaction of a double scatter event generates an $\mathrm{S} 2$ which is below the threshold, such an event is mis-identified as a single scatter interaction. Since in the measured data the energy of an event is computed from the scintillation signal, the average energy of these events is higher than that of true single scatter interactions. ERs are less affected by this detection efficiency, as the typical S2 signals are much larger than the 


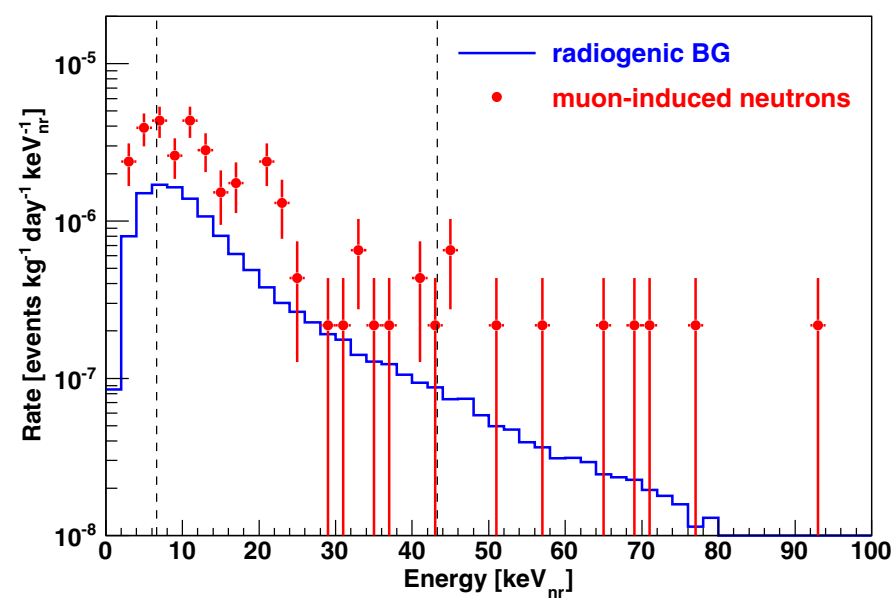

(a) raw Monte Carlo data

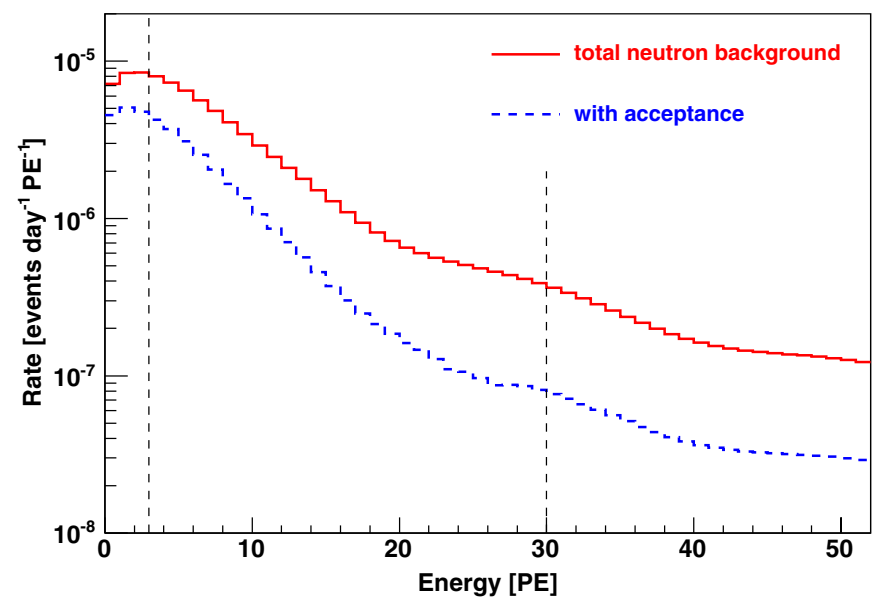

(b) converted to $\mathrm{PE}$, with energy resolution and acceptance

Figure 8. Energy spectra of the NR background due to radiogenic and cosmogenic neutrons predicted for the 2012 WIMP search analysis ( $34 \mathrm{~kg}$ fiducial volume). The prediction takes into account the measured trigger threshold which is the cause of the roll-off at the lowest energies. The bottom plot shows the total NR background on the energy scale converted to PE, with the energy resolution and acceptance of the analysis cuts taken into account. Fluctuations of the light signal for events below the threshold have also been included in this analysis. The thin vertical dashed lines indicate the WIMP search energy region.

threshold value. In order to select single scatter NR interactions in the Monte Carlo data, we apply cuts which are similar to the ones used in the analysis of the measured data.

The energy-dependent acceptance of these cuts was calculated with Monte Carlo simulations, taking into account the $\mathcal{L}_{\text {eff }}$ parametrization [8]. The simulation results cannot be validated via direct comparison with the measured background data due to the very low rate of NRs. Hence, the results were verified by comparing the ratio of single and double scatter events using measured ${ }^{241} \mathrm{AmBe}$ neutron calibration data and a corresponding Monte Carlo simulation, showing excellent agreement within the known uncertainties of $\mathcal{L}_{\text {eff }}$ and the source strength [42]. 
Table 8. NR backgrounds predicted for the 2011 [8] and the 2012 [3] WIMP searches, taking into account the detection efficiency. The NR acceptance after applying an ER discrimination cut based on S2/S1 ratio is not applied as it is not used in the standard Profile Likelihood analysis [43].

\begin{tabular}{|c|c|c|}
\hline $\begin{array}{l}\text { Data release } \\
\text { Live time } \\
\text { Fiducial volume } \\
\text { Energy range }\end{array}$ & $\begin{array}{l}2011[8] \\
100.9 \text { days } \\
48 \mathrm{~kg} \\
8.4-44.6 \mathrm{keV}_{\mathrm{nr}} \\
(4-30 \mathrm{PE})\end{array}$ & $\begin{array}{l}2012[3] \\
224.6 \text { days } \\
34 \mathrm{~kg} \\
6.6-43.3 \mathrm{keV}_{\mathrm{nr}} \\
(3-30 \mathrm{PE})\end{array}$ \\
\hline $\begin{array}{l}\text { Radiogenic neutrons } \\
\text { Cosmogenic neutrons }\end{array}$ & $\begin{array}{l}0.10 \pm 0.02 \text { events } \\
0.21_{-0.12}^{+0.21} \text { events }\end{array}$ & $\begin{array}{l}0.14 \pm 0.02 \text { events } \\
0.34_{-0.17}^{+0.34} \text { events }\end{array}$ \\
\hline tal NR background & $0.31_{-0.11}^{+0.22}$ events & $0.48_{-0.17}^{+0.34}$ events \\
\hline
\end{tabular}

The total NR background rates predicted for the 2011 [7] and the 2012 [8] data analyses, taking into account the detection efficiency, the energy range, and the exposure, are given in table 8 . The contribution from environmental radioactivity (contamination in the rock and concrete of the LNGS laboratory) is at the percent level and has no significant impact on the XENON100 science goals. About 70\% comes from muon-induced neutrons. In future experiments, such as XENON1T [44], this background will be significantly reduced by using a muon veto system. The energy spectra of the background from radiogenic and cosmogenic neutrons predicted for the results published in 2012 are shown in figure 8(a). Figure 8(b) presents the total NR background expected in XENON100, converted to the S1 PE energy scale and corrected for the acceptance due to the analysis cuts, taking into account fluctuations of the light signal for events below the threshold.

\section{Conclusions}

The NR background in the XENON100 experiment, originating from radiogenic and cosmogenic neutrons has been predicted for the XENON100 experiment based on Monte Carlo studies, using a detailed model of the detector and its shield.

The total NR background in the 100.9 days dataset (2011, [8]), which used a fiducial mass of $48 \mathrm{~kg}$ and $(8.4-44.6) \mathrm{keV}_{\mathrm{nr}}$ energy range, is $\left(0.31_{-0.11}^{+0.22}\right)$ events. The detector's energy resolution as well as the active veto efficiency are taken into account here. The prediction for the 224.6 days data used for the WIMP search results of 2012 [3] is $\left(0.48_{-0.17}^{+0.34}\right)$ events. A fiducial target mass of $34 \mathrm{~kg}$ and an energy range of (6.6-43.3) $\mathrm{keV}_{\mathrm{nr}}$ were used for this search.

With the reduced NR acceptance after applying the S2/S1 electronic recoil discrimination cut to define a benchmark WIMP search region, these values translate into $\left(0.11_{-0.04}^{+0.08}\right)$ events, and $\left(0.17_{-0.07}^{+0.12}\right)$ events, respectively. Compared to the total ER background estimates of $(1.7 \pm 0.6)$ events in the 2011 WIMP search, and $(0.8 \pm 0.2)$ events $[8]$ in the result published in 2012, the neutron background does not limit the WIMP search sensitivity of the XENON100 experiment.

The neutron background is even lower at energies above the upper bound used so far. In the (43.3-100) $\mathrm{keV}_{\mathrm{nr}}$ energy range and $48 \mathrm{~kg}$ fiducial target, the contributions from radiogenic and muon-induced neutrons add up to $\left(0.12_{-0.05}^{+0.12}\right)$ background events/year in the full discrimination space. In a $34 \mathrm{~kg}$ target it is only $\left(0.07_{-0.04}^{+0.05}\right)$ events/year, which is about $10 \%$ of the background in the energy range below $30 \mathrm{PE}$. 


\section{Acknowledgments}

We gratefully acknowledge support from NSF, DOE, SNF, UZH, FCT, Région des Pays de la Loire, STCSM, NSFC, DFG, Stichting voor Fundamenteel Onderzoek der Materie (FOM), the Max Planck Society and the Weizmann Institute of Science. We are grateful to LNGS for hosting and supporting XENON100.

\section{References}

[1] Aprile E et al 2012 Astropart. Phys. 35573

[2] Aprile E et al (XENON100 Collaboration) 2012 arXiv:1207.3458

[3] Aprile E et al (XENON100 Collaboration) 2012 Phys. Rev. Lett. 109181301

[4] Aprile E et al (XENON100 Collaboration) 2013 arXiv:1301.6620

[5] Lewin J D and Smith P F 1996 Astropart. Phys. 687 Jungman G, Kamionkowski M and Griest K 2006 Phys. Rep. 267195

[6] Aprile E and Doke T 2010 Rev. Mod. Phys. 822053

[7] Aprile E et al (XENON100 Collaboration) 2010 Phys. Rev. Lett. 105131302

[8] Aprile E et al (XENON100 Collaboration) 2011 Phys. Rev. Lett. 107131302

[9] Aprile E et al (XENON100 Collaboration) 2011 Phys. Rev. D 83082001

[10] Wilson W B et al 1999 SOURCES-4A, Los Alamos Technical Report LA-13539-MS

[11] Kudryavtsev V 2009 Comput. Phys. Commun. 180339

[12] Sulkimo J et al (GEANT4 Collaboration) 2003 Nucl. Instrum. Methods Phys. Res. A 506250

[13] Kish A 2011 PhD Thesis University of Zurich, Switzerland (http://opac.nebis.ch/ediss/20121322.pdf)

[14] Carson M et al 2004 Astropart. Phys. 21667

[15] Mei D, Zhang C and Hime A 2009 Nucl. Instrum. Methods Phys. Res. A 606651

[16] Haffke M et al 2011 Nucl. Instrum. Methods Phys. Res. A 64336

[17] Chu S Y F, Ekstroem L P and Firestone R B 1999 The Lund/LBNL Nuclear Data Search (http://nucleardata. nuclear.lu.se/toi/)

[18] Aprile E et al (XENON100 Collaboration) 2011 Astropart. Phys. 3534

[19] Wulandari H et al 2004 Astropart. Phys. 22313

[20] Aleksan R et al 1989 Nucl. Instrum. Methods Phys. Res. A 274203

[21] Belli P et al 1989 Il Nuovo Cimento A 101959

[22] http://geant4.web.cern.ch/geant4/support/datafiles_origin.shtml

[23] Aglietta M et al (LVD Collaboration) 1998 Phys. Rev. D 5892005

[24] Mei D-M and Hime A 2006 Phys. Rev. D 73053004

[25] Kudryavtsev V A, Spooner N J C and McMillan J E 2003 Nucl. Instrum. Methods Phys. Res. A 505688

[26] Araujo H M et al 2005 Nucl. Instrum. Methods Phys. Res. A 545398

[27] Catalano P G et al 1986 Mem. Soc. Geol. Ital. 35647

[28] Ambrosio M et al (MACRO Collaboration) 1995 Phys. Rev. D 523793

[29] Aglietta M et al (LVD Collaboration) 1998 Phys. Rev. D 58092005

[30] Empl A et al 2012 arXiv:1210.2708

[31] Selvi M et al (LVD Collaboration) 2009 ICRC: Proc. Conf. 31st Int. Cosmic Ray pp 1-4

[32] Adamson P et al (MINOS Collaboration) 2007 Phys. Rev. D 76052003

[33] Agafonova N et al (OPERA Collaboration) 2010 Eur. Phys. J. C 6725

[34] http://geant4.web.cern.ch/geant4/support/proc_mod_catalog/physics_lists/referencePL.shtml

[35] http://geant4.web.cern.ch/geant4/support/proc_mod_catalog/physics_lists/hadronic/QGSP.html

[36] Bogdanov A G et al 2006 IEEE Trans. Nucl. Sci. 53/2 513

[37] Marino M G et al 2007 Nucl. Instrum. Methods Phys. Res. A 582611

[38] Araujo H et al (ZEPLIN-II Collaboration) 2008 Astropart. Phys. 29471

[39] Lindote A et al 2009 Astropart. Phys. 31366

[40] Abe S et al (KamLAND Collaboration) 2010 Phys. Rev. C 81025807

[41] Reichhart L et al (ZEPLIN-III Collaboration) 2013 arXiv:1302.4275

[42] Aprile E et al (XENON100 Collaboration) 2013 arXiv:1304.1427

[43] Aprile E et al (XENON100 Collaboration) 2011 Phys. Rev. D 84052003

[44] Aprile E (XENON1T Collaboration) 2012 arXiv:1206.6288 